\title{
Actitudes hacia las ciencias y logro de aprendizaje en los estudiantes de las instituciones educativas estatales de la provincia de Satipo
}

Rafael M. Cantorín Curty

Email: rcantorin@uncp.edu.pe•Orcid:.http://orcid.org/0000-0002-8971-6042

Henry F. López Cantorín

Email: hlopezcantorin@gmail.com • Orcid: http://orcid.org/0000-0002-8890-7488

Rafael A. Cantorín Benites

Email: raffa26792@gmail.com • Orcid: http://orcid.org/0000-0002-3541-2780

Betzabeth R. Cantorin Benites

Email: betzabeth_cb@hotmail.com •Orcid: http://orcid.org/0000-0003-4865-5375

Diego Crisóstomo Huaringa

Email: mar_dian_1996@hotmail.com・Orcid: http://orcid.org/0000-0003-0711-1785

\section{Resumen}

El estudio se refiere a determinar el índice correlacional entre las actitudes hacia las ciencias y el logro de aprendizaje en las áreas de matemáticas y ciencias naturales; la muestra fueron estudiantes de la institución educativa "Daniel Alcides Carrión" del distrito de Coviriali, provincia de Satipo, región Junín. Tipo de investigación no experimental, nivel descriptivo y diseño correlacional. Concluyendo que los estudiantes se encuentran en el nivel alto en las actitudes hacia las ciencias y sus categorías: cognitivo, emotivo y conductivo; además en las áreas de matemática y ciencias naturales obtuvieron el logro básico (11-13), es decir "el estudiante está en camino de lograr los aprendizajes previstos, para lo cual requiere acompañamiento durante un tiempo razonable para lograrlo". Los índices correlaciones calculados, manifiestan ausencia de correlación en las variables mencionadas.

Palabras clave: Actitud, cognitivo, emocional, conductual, logro básico, matemática, ciencias naturales.

\begin{abstract}
The study refers to determining the correlational index between attitudes towards science and the achievement of learning in the areas of mathematics and natural sciences; the sample were students of the educational institution "Daniel Alcides Carrión" of the district of Coviriali, province of Satipo, Junín region. Type of non-experimental research, descriptive level and correlational design. Concluding that students are at a high level in attitudes towards science and its categories: cognitive, emotional and conductive; In addition, in the areas of mathematics and natural sciences, they obtained the basic achievement (11-13), that is to say "the student is on the way to achieve the expected learning, for which he requires accompaniment for a reasonable time to achieve it". The calculated correlation indices show no correlation in the mentioned variables.
\end{abstract}

Keywords: attitude, cognitive, emotional, behavioral, basic achievement, mathematics, natural sciences. 


\section{Introducción}

El estudio de la Pedagogía Científica y por ende la Educación Científica, son zonas de interés desde hace mucho tiempo, logrando llamar la atención de los investigadores en el área de Humanidades; es así que la actitud hacia las ciencias (matemática y ciencias naturales) es observado a través de la enseñanza y aprendizaje, donde los estudiante consideran que el aprendizaje de los contenidos de las dos áreas: matemática y ciencias naturales son difíciles y aburridos. "Por este motivo, los criterios generales se plantean como ideales que el profesorado tiene que esforzarse en alcanzar mediante la reflexión, la práctica, la interacción y el aprendizaje continuo" (Planas y Alsina; 2009; p. 14).

El interés de los alumnos por el aprendizaje de las ciencias van declinando poco a poco, en algunos casos por la estimulación negativa por parte de los docentes; cuando se realizan las ferias de ciencias los profesores encargan a sus estudiantes a presentar un proyecto, el incumplimiento de esta tarea, los alumnos son merecedores de una nota desaprobatoria (00) y el otro aspecto es el desarrollo y solución de ejercicios y problemas en demasía (tareas para la casa). "Entiendo, además, que el descubrimiento del carácter opinable de algunos aspectos de las matemáticas, y en particular de la estadística, es un aprendizaje crucial que debo tratar, controlando en lo posible el riesgo de que los estudiantes malinterpreten esta indeterminación" (Planas y Alsina; 2007; p. 181).

Los estudiantes del nivel secundario, perciben que el aprendizaje de la matemática y de las ciencias naturales es irrelevante para su vida diaria, sienten que el avance de la ciencia y la tecnología ha generado problemas sociales y ambientales, la ciencia es difícil y es sobre todo hacia los objetos y cosas, por esto y otras razones los alumnos no quieren estudiar las ciencias. Luego, la actitud que muestran los estudiantes hacia la ciencia ha sido explorada y estudiada en otros contextos, en nuestra región hay una escasez de investigaciones.

Las actitudes son juicios evaluativos o medibles formados en cada uno de las personas, considerando luego la actitud como un rasgo hipotético. La evaluación y la decisión subsecuente dependen en cada individuo, el conocimiento, los sentimientos y las experiencias permanecen en las personas; en general, las actitudes una vez establecidas en los sujetos permanecen indefinidamente.

Cofer y Appley (2007) manifiestan "cuando existen actitudes positivas y negativas hacia un mismo objeto, hay conflicto y tensión (por definición, las actitudes indican reacciones afectivas hacia sus referentes" (p. 385). La actitud científica comprende: componentes cognitivos, emocionales y conductuales, lo que lleva a desarrollar un comportamiento en particular.

Vásquez y Manassero (2001) definen la actitud similar a la psicología social, es decir, lo aceptan como una motivación en las categorías cognitivo y conductual, destacando la emotividad desde un punto de vista favorable o desfavorable del individuo. Para Pozo y Gómez (2001) las actitudes son las predisposiciones cercanas o de alejamiento con respecto a algo, que se descifran en tendencias o recelos que definen el comportamiento de los individuos.

Entiendo el aprendizaje como un proceso permanente de interacciones que resulta en la adaptación y/o acomodación de la persona a un medio altamente cambiante y que hace posible un continuo mejoramiento de las condiciones de vida. De esta forma, los conocimientos formalizados, construidos en este proceso, serán un subproducto tan plástico y cambiante como lo son las interrelaciones que lo hicieron posible. De ser comprendida como transmisión de conocimientos, de estar centrada en la enseñanza, de tener como agente fundamental 
al aprendiz (estudiante, como destinatario de su esfuerzo y de sus conocimientos) y al docente como facilitador-orientador del proceso personal de aprendizaje de sus pupilos.

MINEDU (1999) "Es por ello que la persona de cada alumno cobra especial importancia por sobre los contenidos a ser enseñados, al punto que se exige priorizar sus necesidades y motivaciones, sus capacidades y habilidades, sus valores y actitudes" (p. 10). Que el estudiante pueda conseguirlo supone no sólo que haya logrado un nivel madurativo necesario, sino que existen las condiciones como una forma cualitativamente distinta de interrelación social. Todo cambio en el ser humano está genéticamente condicionado, sólo si el medio lo permite y da garantías para un buen desarrollo va a terminar por producir un individuo apto para el máximo de supervivencia, con un gran respeto por el otro, por sí mismo, por un ser superior y por naturaleza.

Problema general: ¿cuál es la relación de las actitudes hacia las ciencias y el logro de aprendizaje en los estudiantes de educación secundaria de las instituciones educativas estatales de la provincia de Satipo? Problemas especificos: (a) ¿cuál es la relación de las actitudes hacia las ciencias y el logro de aprendizaje en matemática de los estudiantes de educación secundaria de las instituciones educativas estatales de la provincia de Satipo? (b) ¿cuál es la relación de las actitudes hacia las ciencias y el logro de aprendizaje en las ciencias naturales de los estudiantes de educación secundaria de las instituciones educativas estatales de la provincia de Satipo? (c) ¿cuál es la relación de las categorías de las actitudes hacia las ciencias y el logro de aprendizaje en los estudiantes de educación secundaria de las instituciones educativas estatales de la provincia de Satipo?.

"Los objetivos sirven para evaluar la investigación en sus propósitos o alcances. Todo trabajo de investigación es evaluado por el logro de los objetivos, de ahí la importancia que estén bien escritos y formulados" (Toro y Parra, 2010, p. 182). Objetivo general: Determinar la relación de las actitudes hacia las ciencias y el logro de aprendizaje de los estudiantes de educación secundaria de las instituciones educativas estatales de la provincia de Satipo. Objetivos especificos: (a) Identificar y describir la relación de las actitudes hacia las ciencias y el logro de aprendizaje en matemática en los estudiantes de educación secundaria de las instituciones educativas estatales de la provincia de Satipo; (b) Identificar y describir la relación de las actitudes hacia las ciencias y el logro de aprendizaje en las ciencias naturales en los estudiantes de educación secundaria de las instituciones educativas estatales de la provincia de Satipo; (c) Identificar y describir la relación de las categorías de las actitudes hacia las ciencias y el logro de aprendizaje en los estudiantes de educación secundaria de las instituciones educativas estatales de la provincia de Satipo.

Hipótesis general: La relación de las actitudes hacia las ciencias y el logro de aprendizaje de los estudiantes de educación secundaria de las instituciones educativas estatales de la provincia de Satipo, es moderada positiva. Hipótesis especificas: (a) La relación de las actitudes hacia las ciencias y el logro de aprendizaje en matemática en los estudiantes de educación secundaria de las instituciones educativas estatales de la provincia de Satipo es moderada positiva; (b) La relación de las actitudes hacia las ciencias y el logro de aprendizaje en las ciencias naturales en los estudiantes de educación secundaria de las instituciones educativas estatales de la provincia de Satipo es moderada positiva; (c) La relación de las categorías de las actitudes hacia las ciencias y el logro de aprendizaje en los estudiantes de educación secundaria de las instituciones educativas estatales de la provincia de Satipo es moderada positiva. 


\section{Materiales y métodos}

La investigación es de tipo descriptiva. Los estudios descriptivos miden las variables, la descripción puede ser más o menos profunda, pero en cualquier caso se basa en la medición de uno o más atributos de las variables descritas: actitudes y logro de aprendizaje (Toro y Parra; 2010).

El diseño utilizado fue el transeccional correlacional. "En estos diseños lo que se mide es la relación entre variables en un tiempo determinado" (Toro y Parra; 2010; p. 277). Se emplearon los instrumentos: Cuestionario de actitudes hacia las ciencias y Registro de notas del primer bimestre.

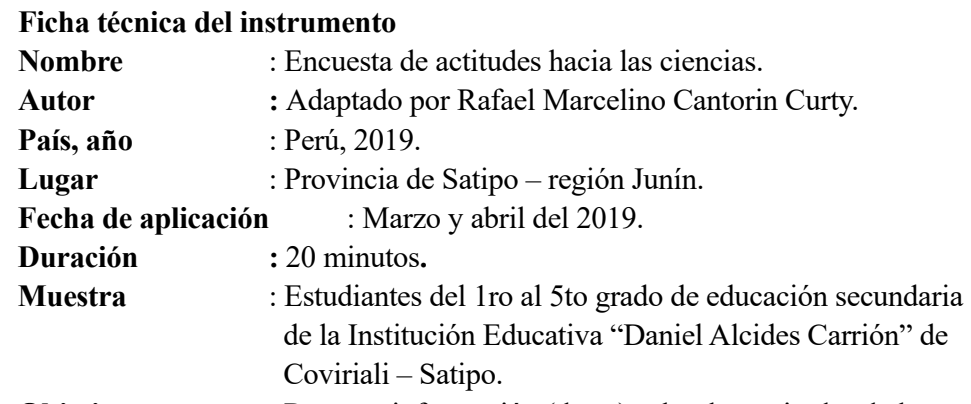

Objetivo : : Recoger información (datos) sobre las actitudes de los alumnos hacia las ciencias (Matemática y Ciencias Naturales).

Características : Psicométrica, utiliza las respuestas de la escala Likert.

Respuesta positiva : Totalmente en desacuerdo (1), en desacuerdo (2), indiferente (3), de acuerdo (4) y totalmente de acuerdo (5).

Respuesta negativa : Totalmente en desacuerdo (5), en desacuerdo (4), indiferente (3), de acuerdo (2) y totalmente de acuerdo (1).

Dimensiones : Cognitivo, ítems de respuestas positivas: 2, 16,17 y 19.

Ítems de respuestas negativas: 4,12 y 20 .

Emotivo, ítems de respuestas positivas: 1, 5, 10 y 15.

Ítems de respuestas negativas: 9 y 11.

Conductivo, ítems de respuestas positivas: 3, 6, 13 y 18 .

Ítems de respuestas negativas: 7, 8 y 14.

\section{Resultados}

El trabajo de investigación cuyo título es: Actitudes hacia las ciencias y logro de aprendizaje en los estudiantes de las instituciones educativas estatales de la provincia de Satipo, los datos fueron recopilados a través de una encuesta de actitudes hacia las ciencias (Matemática y Ciencias Naturales), compuesta por 20 ítems y el logro de aprendizaje a través de sus notas del primer bimestre. La encuesta de actitudes tiene 5 respuestas (escala Likert): totalmente en desacuerdo, en desacuerdo, indiferente, de acuerdo y totalmente de acuerdo; y en cuanto se refiere a la otra variable logro de aprendizaje, se tiene en puntuaciones en la escala vigesimal (de 00 a 20), con sus equivalentes en la escala cualitativa, como son: logro inicial, logro básico, logro satisfactorio y logro muy satisfactorio. 


\section{Variable actitudes hacia las ciencias}

Cuadro 1.

Actitudes hacia las ciencias.

\begin{tabular}{|c|c|c|c|}
\hline Respuestas & $\mathbf{N}^{\circ}$ Estud. & $\mathbf{N}^{\circ}$ Rptas. & $(\%)$ \\
\hline Totalmente en desacuerdo & 12 & 72 & $16 \%$ \\
\hline En desacuerdo & 12 & 76 & $16 \%$ \\
\hline Indiferente & 5 & 49 & $6 \%$ \\
\hline De acuerdo & 22 & 126 & $29 \%$ \\
\hline Totalmente de acuerdo & 26 & 216 & $34 \%$ \\
\hline & 77 & 539 & $100 \%$ \\
\hline
\end{tabular}

Figura 1.

Actitudes hacia las ciencias.

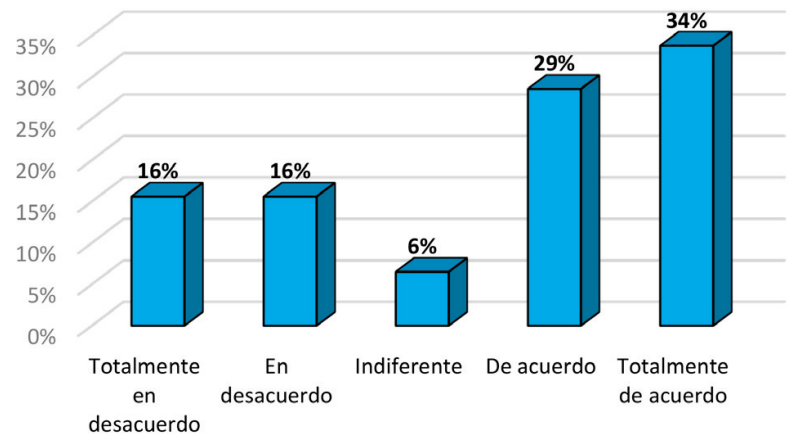

En resumen se tienen 12 estudiantes (16\%) que están "totalmente en desacuerdo" y "en desacuerdo" al percibir 72 y 76 respuestas respectivamente; 5 estudiantes (6\%) que son “indiferentes” al percibir 49 respuestas; 22 estudiantes (29\%) que están “de acuerdo” al percibir 126 respuestas; y 26 estudiantes (34\%) que están "totalmente de acuerdo" al percibir 216 respuestas. Concluyendo que la mayoría de los alumnos de la organización educativa "Daniel Alcides Carrión" de Coviriali están totalmente de acuerdo con las actitudes que tiene hacia las ciencias.

Cuadro 2.

Actitudes hacia las ciencias, en la dimensión cognitiva.

\begin{tabular}{lccc}
\hline Respuestas & $\mathbf{N}^{\circ}$ Estud. & $\mathbf{N}^{\circ}$ Rptas. & $\mathbf{( \% )}$ \\
\hline Totalmente en desacuerdo & 10 & 72 & $13 \%$ \\
En desacuerdo & 11 & 76 & $14 \%$ \\
Indiferente & 7 & 49 & $9 \%$ \\
De acuerdo & 18 & 126 & $24 \%$ \\
Totalmente de acuerdo & 31 & 216 & $40 \%$ \\
\hline & & $\mathbf{5 3 9}$ & $\mathbf{1 0 0 \%}$ \\
\hline
\end{tabular}


Figura 2.

Actitudes hacia las ciencias, en la dimensión cognitiva.

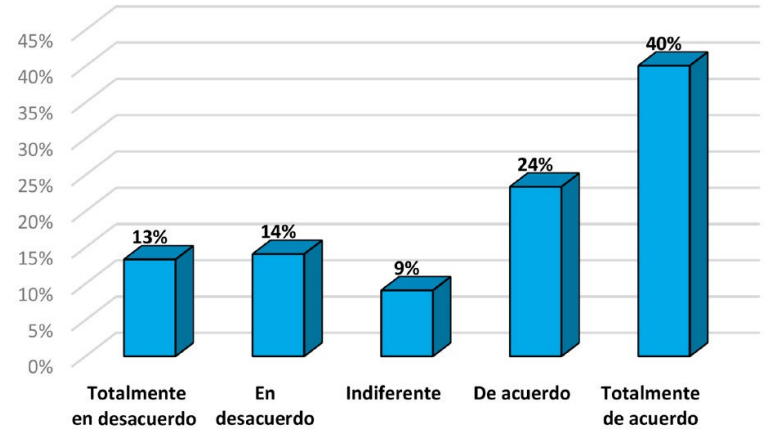

En resumen se tienen 10 estudiantes (13\%) que están "totalmente en desacuerdo" al percibir 72 respuestas; 11 estudiantes (14\%) que están "en desacuerdo” al percibir 76 respuestas; 7 estudiantes (9\%) que son "indiferentes" al percibir 49 respuestas; 18 estudiantes $(24 \%)$ que están “de acuerdo” al percibir 126 respuestas; y 31 estudiantes (40\%) que están “totalmente de acuerdo" al percibir 216 respuestas. Concluyendo que la mayoría de los alumnos de la organización educativa "Simón Bolívar" de Coviriali están totalmente de acuerdo con las actitudes que tiene hacia las ciencias.

Cuadro 3.

Actitudes hacia las ciencias, en la dimensión emotiva.

\begin{tabular}{lccc}
\hline Respuestas & $\mathbf{N}^{\circ}$ Estud. & $\mathbf{N}^{\circ}$ Rptas. & $\mathbf{( \% )}$ \\
\hline Totalmente en desacuerdo & 14 & 82 & $18 \%$ \\
En desacuerdo & 13 & 76 & $17 \%$ \\
Indiferente & 4 & 23 & $5 \%$ \\
De acuerdo & 18 & 111 & $23 \%$ \\
Totalmente de acuerdo & 28 & 170 & $37 \%$ \\
\hline & & $\mathbf{4 6 2}$ & $\mathbf{1 0 0 \%}$ \\
\hline
\end{tabular}

Figura 3.

Actitudes hacia las ciencias, en la dimensión emotiva.

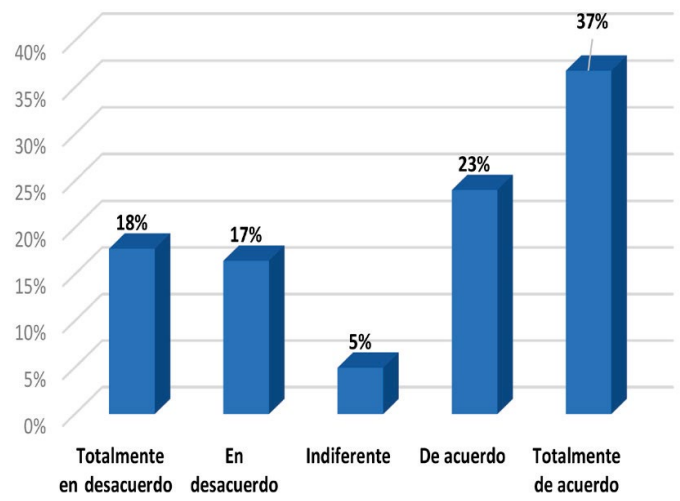


En resumen se tienen 14 estudiantes (18\%) que están “totalmente en desacuerdo" al percibir 82 respuestas; 13 estudiantes (17\%) que están "en desacuerdo” al percibir 76 respuestas; 4 estudiantes (5\%) que son "indiferentes" al percibir 23 respuestas; 18 estudiantes $(23 \%)$ que están "de acuerdo" al percibir 111 respuestas; y 28 estudiantes (37\%) que están "totalmente de acuerdo" al percibir 170 respuestas. Concluyendo que la mayoría de los alumnos de la organización educativa "Simón Bolívar" de Coviriali están totalmente de acuerdo con las actitudes que tiene hacia las ciencias.

Cuadro 4.

Actitudes hacia las ciencias, en la dimensión conductiva.

\begin{tabular}{|c|c|c|c|}
\hline Respuestas & $\mathrm{N}^{\circ}$ Estud. & $\mathrm{N}^{\circ}$ Rptas. & $(\%)$ \\
\hline Totalmente en desacuerdo & 12 & 83 & $15 \%$ \\
\hline En desacuerdo & 11 & 79 & $15 \%$ \\
\hline Indiferente & 4 & 30 & $6 \%$ \\
\hline De acuerdo & 30 & 207 & $38 \%$ \\
\hline Totalmente de acuerdo & 20 & 140 & $26 \%$ \\
\hline & 77 & 539 & 100 \\
\hline
\end{tabular}

Figura 4.

Actitudes hacia las ciencias, en la dimensión conductiva.

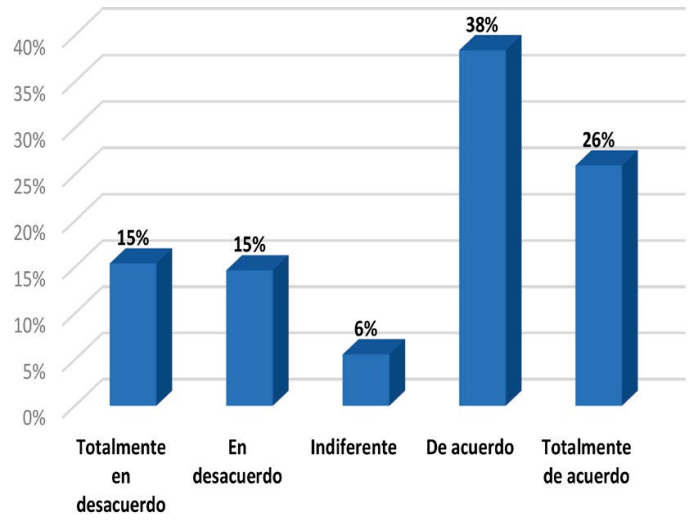

En resumen se tienen 12 y 11 estudiantes (15\%) que están “totalmente en desacuerdo" y "en desacuerdo" al percibir 83 y 79 respuestas respectivamente; 4 estudiantes (6\%) que son “indiferentes" al percibir 30 respuestas; 30 estudiantes (28\%) que están “de acuerdo” al percibir 207 respuestas; y 20 estudiantes (26\%) que están “totalmente de acuerdo" al percibir 140 respuestas. Concluyendo que la mayoría de los alumnos de la organización educativa "Simón Bolívar" de Coviriali están de acuerdo con las actitudes que tiene hacia las ciencias. 
Cuadro 5.

Resumen de las actitudes hacia las ciencias.

\begin{tabular}{|c|c|c|c|c|c|c|}
\hline \multirow{2}{*}{ Respuestas } & \multicolumn{2}{|c|}{ Cognitivo } & \multicolumn{2}{|c|}{ Emotivo } & \multicolumn{2}{|c|}{ Conductivo } \\
\hline & Rptas. & $(\%)$ & Rptas. & $(\%)$ & Rptas. & $(\%)$ \\
\hline Totalmente en desacuerdo & 72 & $13 \%$ & 82 & $18 \%$ & 83 & $15 \%$ \\
\hline En desacuerdo & 76 & $14 \%$ & 76 & $17 \%$ & 79 & $15 \%$ \\
\hline Indiferente & 49 & $9 \%$ & 23 & $5 \%$ & 30 & $6 \%$ \\
\hline De acuerdo & 126 & $24 \%$ & 111 & $23 \%$ & 207 & $38 \%$ \\
\hline Totalmente de acuerdo & 216 & $40 \%$ & 170 & $37 \%$ & 140 & $26 \%$ \\
\hline Total & 539 & $100 \%$ & 462 & $100 \%$ & 539 & $100 \%$ \\
\hline
\end{tabular}

Figura 5.

Resumen de las actitudes hacia las ciencias.

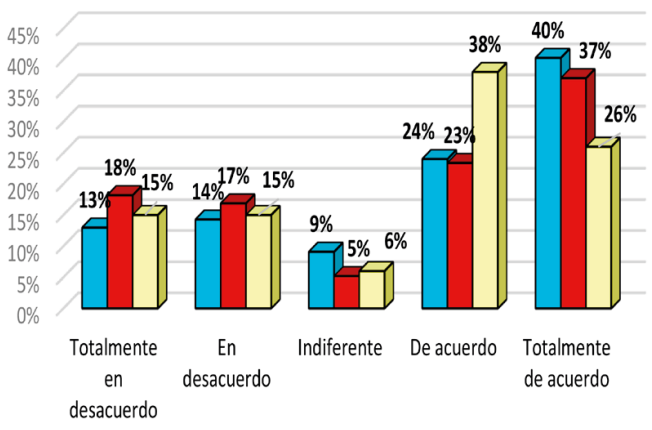

$\square$ Cogntivo $\square$ Emotivo $\square$ Conductivo

Se percibe en la respuesta "totalmente en desacuerdo" en la dimensión cognitiva 13\%; dimensión emotiva 18\%; dimensión conductiva 15\%; también en la respuesta "en desacuerdo" en la dimensión cognitiva 14\%; dimensión emotiva 17\%; dimensión conductiva 15\%; además en la respuesta "indiferente" en la dimensión cognitiva 9\%; dimensión emotiva 5\%; dimensión conductiva 6\%; asimismo en la respuesta "de acuerdo" en la dimensión cognitiva 24\%; dimensión emotiva 23\%; dimensión conductiva 38\%; y en la respuesta "totalmente de acuerdo" en la dimensión cognitiva 40\%; dimensión emotiva $37 \%$; dimensión conductiva $26 \%$.

Cuadro 6.

Niveles de las actitudes hacia las ciencias.

\begin{tabular}{|c|c|c|}
\hline Niveles & $\mathbf{N}^{\circ}$ Estud. & $(\%)$ \\
\hline Muy bajo & 00 & $00 \%$ \\
\hline Bajo & 00 & $00 \%$ \\
\hline Medio & 14 & $18 \%$ \\
\hline Alto & 63 & $82 \%$ \\
\hline Muy alto & 00 & $00 \%$ \\
\hline & 77 & $100 \%$ \\
\hline
\end{tabular}


Figura 6.

Niveles de las actitudes hacia las ciencias.

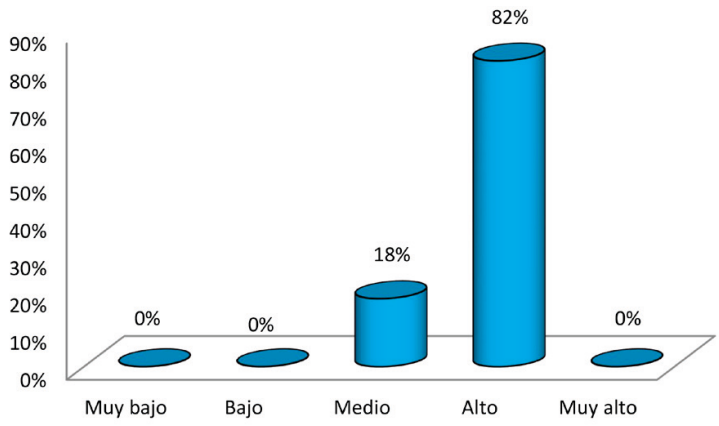

En resumen se tienen 14 alumnos (18\%) que están en el "nivel medio” y 63 estudiantes (82\%) que están en el "nivel alto”. Concluyendo que la mayoría de los alumnos de la organización educativa "Simón Bolívar" de Coviriali están en el nivel alto en las actitudes que tienen hacia las ciencias.

Cuadro 7.

Niveles de las actitudes hacia las ciencias, en la dimensión cognitiva.

\begin{tabular}{|c|c|c|}
\hline Niveles & $\mathrm{N}^{\circ}$ Estud. & $(\%)$ \\
\hline Muy bajo & 00 & $00 \%$ \\
\hline Bajo & 00 & $00 \%$ \\
\hline Medio & 22 & $29 \%$ \\
\hline Alto & 55 & $71 \%$ \\
\hline Muy alto & 00 & $00 \%$ \\
\hline & 77 & $100 \%$ \\
\hline
\end{tabular}

Figura 7.

Niveles de las actitudes hacia las ciencias, en la dimensión cognitiva.

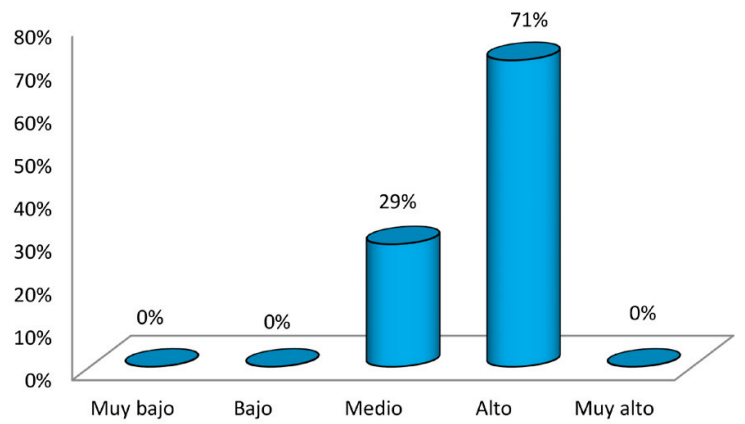


En resumen se tienen 22 alumnos (29\%) que están en el "nivel medio" y 55 estudiantes (71\%) que están en el "nivel alto”. Concluyendo que la mayoría de los alumnos de la organización educativa "Simón Bolívar" de Coviriali están en el nivel alto en las actitudes que tienen hacia las ciencias.

\section{Cuadro 8.}

Niveles de las actitudes hacia las ciencias, en la dimensión emotiva.

\begin{tabular}{|c|c|c|}
\hline Niveles & $\mathrm{N}^{\circ}$ Estud. & Porcentaje \\
\hline Muy bajo & 00 & $00 \%$ \\
\hline Bajo & 1 & $1 \%$ \\
\hline Medio & 30 & $39 \%$ \\
\hline Alto & 46 & $60 \%$ \\
\hline Muy alto & 00 & $00 \%$ \\
\hline Total & 77 & $100 \%$ \\
\hline
\end{tabular}

Figura 8 .

Niveles de las actitudes hacia las ciencias, en la dimensión cognitiva.

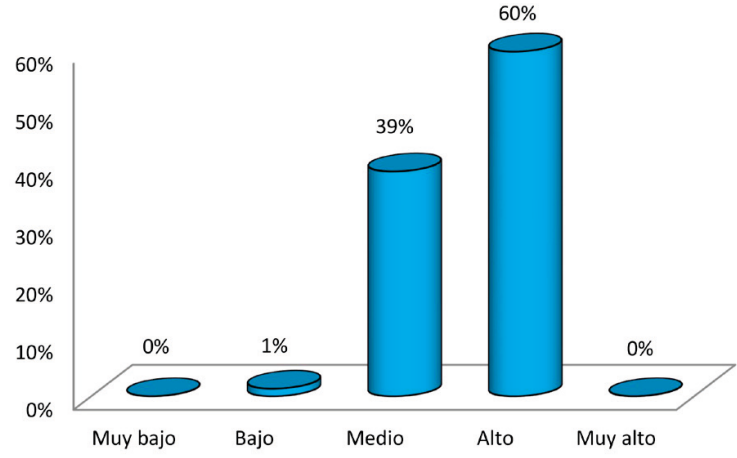

En resumen se tienen un estudiante que está en el "nivel bajo"; 30 alumnos (39\%) que están en el "nivel medio” y 46 estudiantes (60\%) que están en el "nivel alto”. Concluyendo que la mayoría de los alumnos de la organización educativa "Simón Bolívar" de Coviriali están en el nivel alto en las actitudes que tienen hacia las ciencias.

\section{Cuadro 9.}

Niveles de las actitudes hacia las ciencias, en la dimensión conductiva.

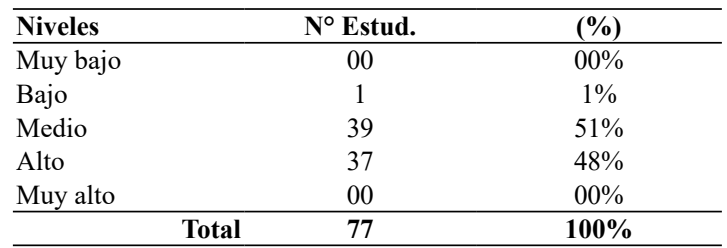




\section{Figura 9.}

Niveles de las actitudes hacia las ciencias, en la dimensión conductiva.

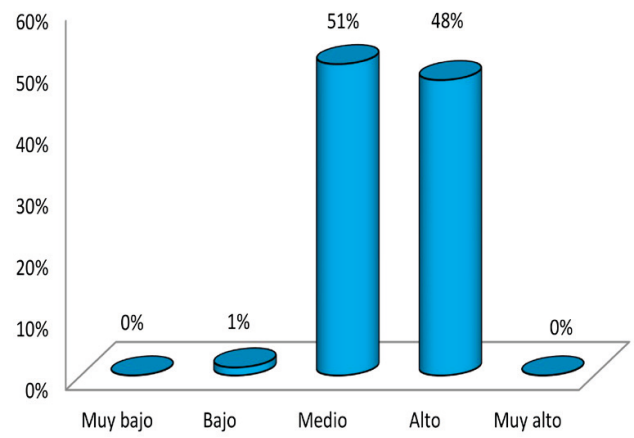

En resumen se tienen un estudiante que está en el "nivel bajo"; 39 alumnos (51\%) que están en el "nivel medio" y 37 estudiantes (48\%) que están en el "nivel alto”. Concluyendo que la mayoría de los alumnos de la organización educativa "Simón Bolívar" de Coviriali están en el nivel medio en las actitudes que tienen hacia las ciencias.

Cuadro 10.

Resumen de los niveles de las actitudes hacia las ciencias.

\begin{tabular}{|c|c|c|c|c|c|c|}
\hline \multirow{2}{*}{ Niveles } & \multicolumn{2}{|c|}{ Cognitivo } & \multicolumn{2}{|c|}{ Emotivo } & \multicolumn{2}{|c|}{ Conductivo } \\
\hline & $\mathbf{N}^{\circ}$ & $(\%)$ & $\mathbf{N}^{\circ}$ & $(\%)$ & $\mathbf{N}^{\circ}$ & $(\%)$ \\
\hline Muy bajo & 00 & $00 \%$ & 00 & $00 \%$ & 00 & $00 \%$ \\
\hline Bajo & 00 & $00 \%$ & 1 & $1 \%$ & 1 & $1 \%$ \\
\hline Medio & 22 & $29 \%$ & 30 & $39 \%$ & 39 & $51 \%$ \\
\hline Alto & 55 & $71 \%$ & 46 & $60 \%$ & 37 & $48 \%$ \\
\hline Muy alto & 00 & $00 \%$ & 00 & $00 \%$ & 00 & $00 \%$ \\
\hline & 77 & $100 \%$ & 77 & $100 \%$ & 77 & $100 \%$ \\
\hline
\end{tabular}

Figura 10.

Resumen de los niveles de las actitudes hacia las ciencias.

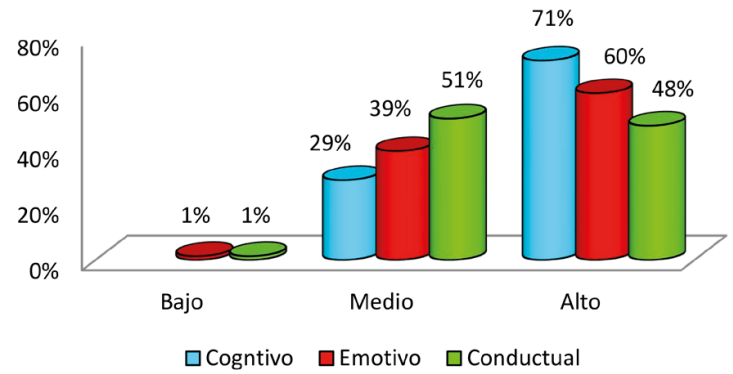

Se observa en el "nivel bajo" en la dimensión emotiva y conductiva 1\%; también en el "ni- 
vel medio" en la dimensión cognitiva $29 \%$; dimensión emotiva $39 \%$; dimensión conductiva $51 \%$; además en el "nivel alto" en la dimensión cognitiva $71 \%$; dimensión emotiva $60 \%$; dimensión conductiva $48 \%$.

\section{Variable logro académico}

Cuadro 11.

Logro académico en ciencias.

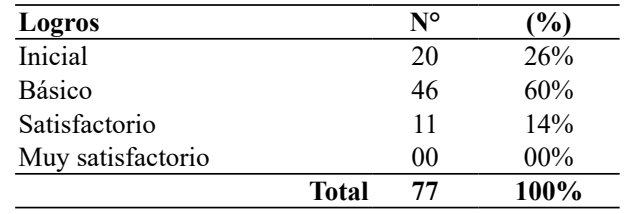

Figura 11.

Logro académico en ciencias.

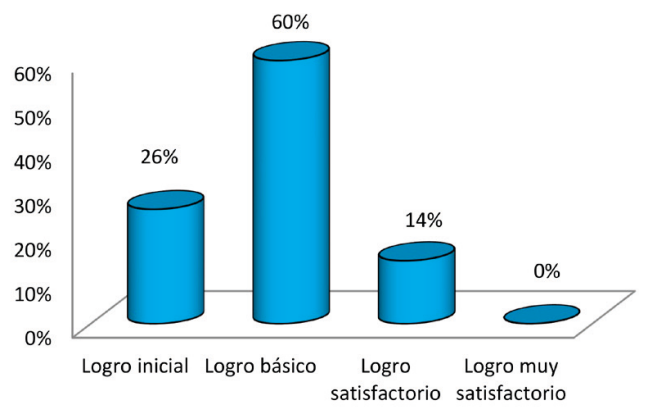

Se observa 20 estudiantes (26\%) que están "logro inicial”; 46 estudiantes (60\%) que están "logro básico”; y 11 estudiantes (14\%) están en "logro satisfactorio”. Concluyendo que la mayoría de los alumnos de la organización educativa "Simón Bolívar" de Coviriali 'califican en el logro básico.

Cuadro 12.

Logro académico en Matemática.

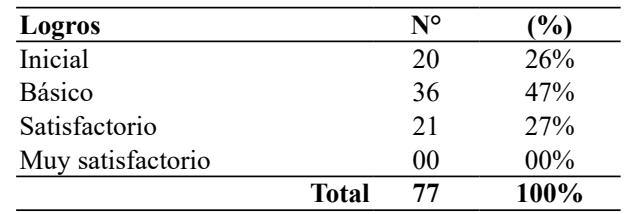


Figura 12.

Logro académico en Matemática.

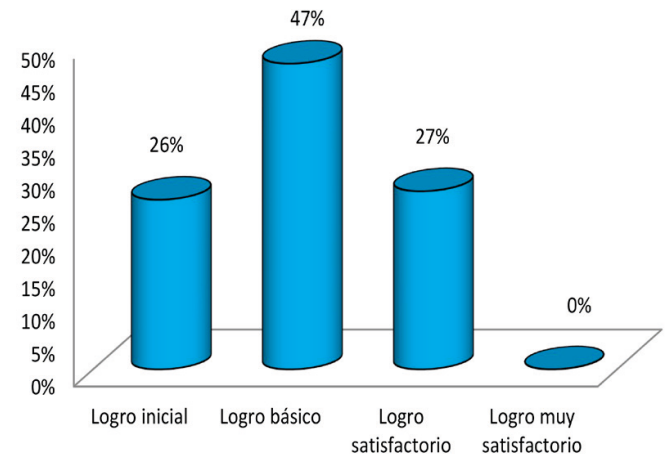

Se observa 20 estudiantes (26\%) que están “logro inicial”; 36 estudiantes (47\%) que están “logro básico”; y 21 estudiantes (27\%) están en “logro satisfactorio”. Concluyendo que la mayoría de los alumnos de la organización educativa "Simón Bolívar" de Coviriali califican en el logro básico.

Cuadro 13.

Logro académico en Ciencias Naturales.

\begin{tabular}{|c|c|c|}
\hline Logros & $\mathbf{N}^{\circ}$ & $(\%)$ \\
\hline Inicial & 21 & $27 \%$ \\
\hline Básico & 29 & $38 \%$ \\
\hline Satisfactorio & 27 & $35 \%$ \\
\hline Muy satisfactorio & 00 & $00 \%$ \\
\hline & 77 & $100 \%$ \\
\hline
\end{tabular}

Figura 13.

Logro académico en Ciencias Naturales.

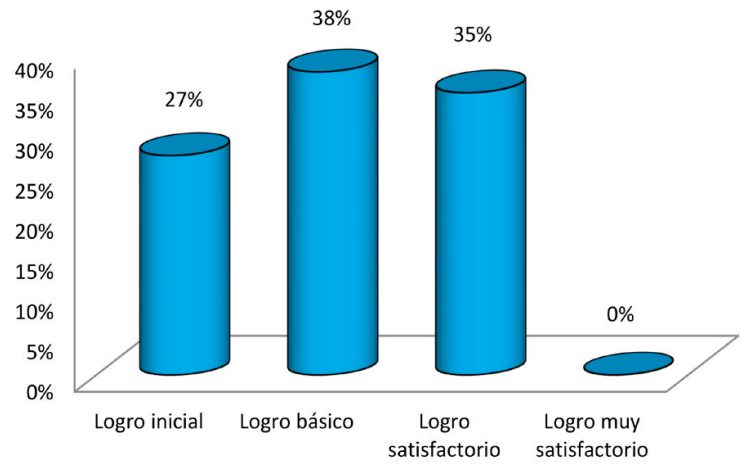


Se observa 21 estudiantes (27\%) que están "logro inicial”; 29 estudiantes (38\%) que están "logro básico"; y 27 estudiantes (35\%) están en "logro satisfactorio”. Concluyendo que la mayoría de los alumnos de la organización educativa "Simón Bolívar" de Coviriali califican en el logro básico.

Cuadro 14.

Logro académico en Matemática y Ciencias Naturales.

\begin{tabular}{|c|c|c|c|c|}
\hline \multirow{2}{*}{ Logros } & \multicolumn{2}{|c|}{ Matemática } & \multicolumn{2}{|c|}{ C. Naturales } \\
\hline & $\mathbf{N}^{\circ}$ & $(\%)$ & $\mathbf{N}^{\circ}$ & $(\%)$ \\
\hline Inicial & 20 & $26 \%$ & 21 & $27 \%$ \\
\hline Básico & 36 & $47 \%$ & 29 & $38 \%$ \\
\hline Satisfactorio & 21 & $27 \%$ & 27 & $35 \%$ \\
\hline Muy satisfactorio & 00 & $00 \%$ & 00 & $00 \%$ \\
\hline & 77 & $100 \%$ & 77 & $100 \%$ \\
\hline
\end{tabular}

Figura 14.

Logro académico en Matemática y Ciencias Naturales.

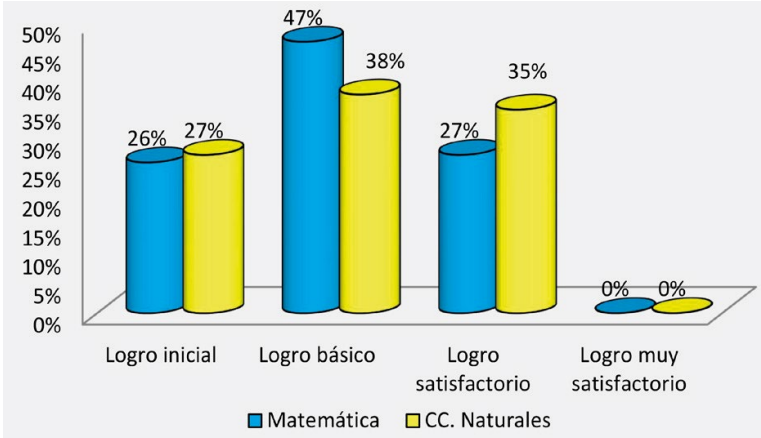

Se observa en matemática y ciencias naturales $26 \%$ y $27 \%$ que están en el "logro inicial”; $47 \%$ y 38\% están “logro básico”; $27 \%$ y 35\% están en "logro satisfactorio” respectivamente. Concluyendo que la mayoría de los alumnos de la organización educativa "Simón Bolívar" de Coviriali 'califican en el logro básico.

Sean las hipótesis:

La relación de las actitudes hacia las ciencias y el logro de aprendizaje de los estudiantes de educación secundaria de las instituciones educativas estatales de la provincia de Satipo, es moderado positivo.

\section{Hipótesis estadísticas}

Ho: No existe relación entre las actitudes hacia las ciencias y sus categorías con la variable logro de aprendizaje en matemática y ciencias naturales en los estudiantes de educación secundaria de las instituciones educativas estatales de la provincia de Satipo.

$$
\text { Ho: } r=0
$$

Ha: Existe relación moderada positiva entre las actitudes hacia las ciencias y sus categorías con el logro de aprendizaje en matemática y ciencias naturales en los estudiantes de educación secundaria de las instituciones educativas estatales de la provincia de Satipo.

Ha: $r>0$ 
Sean los resultados:

\begin{tabular}{lccc}
\hline & Ciencias & Matemática & Ciencias naturales \\
\hline Actitud hacia las ciencias & $-0,024$ & $-0,094$ & 0,056 \\
Cognitivo & $-0,014$ & $-0,083$ & 0,059 \\
Emotivo & $-0,014$ & $,-0,063$ & 0,041 \\
Conductivo & $-0,026$ & $-0,052$ & 0,014 \\
\hline
\end{tabular}

De lo percibido en las correlaciones, según Pearson, se acepta la hipótesis nula y se rechaza las hipótesis alternas; es decir, no existe relación entre las actitudes hacia las ciencias y sus categorías con la variable logro de aprendizaje en matemática y ciencias naturales en los estudiantes de educación secundaria de las instituciones educativas estatales de la provincia de Satipo.

Además podemos inferir que pese a los resultados porcentuales de los niveles de las actitudes, "nivel "alto" y los resultados del logro académico en matemática y ciencias naturales, "logro básico", cuantitativamente estarían sus puntuaciones entre 11 y 13; la ausencia de relación estaría reflejando que no existe una compatibilidad entre estas dos variables o que los profesores enseñan por enseñar (no existe motivación o actitud hacia el conocimiento de las ciencias). Este impase obtenido en el estudio nos sugiere preparar talleres de capacitación en estrategias de aprendizaje.

\section{Discusión}

Con respecto al estudio realizado, se eligió a 77 estudiantes del centro educativo "Daniel Alcides Carrión” del distrito de Coviriali, provincia de Satipo, región Junín con la técnica muestral no probabilística al igual que Pietro y Vera (2008) quienes trabajaron con 908 estudiantes elegidos con la misma técnica muestral. Diferimos con los investigadores mencionados en el tipo y diseño de estudio, pues, ellos realizaron un estudio descriptivo comparativo, y la investigación motivo de estudio utiliza el tipo de investigación descriptivo con un diseño transeccional-correlacional.

Pietro y Vera muestran una actitud positiva de los estudiantes hacia la ciencia, además encontró diferencias significativas en la jornada de estudio, siendo el turno tarde la que presenta mayor puntuación promedio, en nuestro estudio desarrollado concluimos que también los alumnos tienen una actitud hacia las ciencias categorizado en el nivel alto (82\%) frente a un nivel medio de 18\%; diferenciándonos con dichos investigadores al no realizar el control de los turnos de clase, pues la mencionada institución educativa solo contaba con un solo turno..

Cóndor y Miranda (2017) concluyen: las actitudes de los alumnos hacia la Física son positivas al interior de las categorías de estudio, cognoscitivo, emocional y actitudinal; según genero las actitudes de los estudiantes son positivas, distinguiéndose en cantidad el sexo masculino; del mismo modo encontramos en el estudio realizado el nivel alto en las dimensiones cognitiva (71\%) y emotivo (60\%), siendo diferente en la dimensión conductual donde logran el mayor porcentaje en el nivel medio (51\%) estando cerca el nivel alto con $48 \%$.

Asimismo Cóndor y Miranda utilizan la prueba chí cuadrada para demostrar lo significativo de la relación entre los conocimientos básicos del área y las actitudes, afirmando el vínculo de la teoría del aprendizaje con las actitudes. En la pesquisa desarrollada se utiliza la correlación de Pearson para determinar la relación y aceptar la hipótesis de investigación (la relación de las actitudes hacia las ciencias y el logro de aprendizaje de los estudiantes de 
educación secundaria de las instituciones educativas estatales de la provincia de Satipo es moderada positiva), sin embargo se obtuvo índices correlaciónales negativos $\left(\mathbf{r}_{\mathrm{A}-\mathrm{C}}=-0,024 \mathrm{y}\right.$ $\left.\mathbf{r}_{\mathrm{A}-\mathrm{M}}=-0,094\right)$ entonces estas variables de actitudes hacia las ciencias y logro de aprendizaje aparentemente muestran relaciones positivas en otros estudios, pero en nuestra realidad no lo es "lo que se conoce con el nombre de correlaciones espurias" Toro y Parra $(2010 ; 248)$.

\section{Conclusiones}

El índice correlacional de las actitudes hacia las ciencias y el logro de aprendizaje de los estudiantes de las organizaciones educativas de la provincia de Satipo, es nula, no logrando el objetivo general, evaluada con la hipótesis de trabajo (hipótesis alterna), categorizándose como correlación espuria; es decir, el conocimiento, los sentimientos y las experiencias depende de cada estudiante y estaría demostrándose la aversión que tienen los estudiantes hacia las ciencias (matemática, física y química).

La relación de las actitudes hacia las ciencias y el logro de aprendizaje de los estudiantes de las instituciones educativas del nivel secundario de la provincia de Satipo, es nula, no logrando los objetivos específicos, evaluados con la hipótesis específicas de trabajo (hipótesis alterna), categorizándose también como una correlación espuria; es decir los conocimientos, los sentimientos y las experiencias depende de cada alumno y la actitud una vez establecida en él permanece indefinidamente.

\section{Referencias}

Cofer, C. y Appley, M. (2007). Psicología de la motivación. Teoría e investigación. (2da. Edición). México: Trillas.

Cóndor, G. y Miranda, W. (2017). Actitudes hacia la fisica en estudiantes del quinto grado de educación secundaria de instituciones educativas de gestión pública de El Tambo. Tesis de licenciatura. Huancayo-Junín: Universidad Nacional del Centro del Perú.

Hernández, N. (2015). Actitudes hacia la ciencia en estudiantes de $4^{\circ}$ grado de secundaria del distrito de San Juan de Lurigancho, Lima. Tesis de maestría. Lima: Universidad Peruana Cayetano Heredia.

Ministerio de Educación - MINEDU (1999). Bachillerato peruano. Lima: Imprenta del Ministerio de Educación del Perú.

Pietro, L. y Vera, A. (2008). Actitudes hacia la ciencia en estudiantes de secundaria. Bogotá-Colombia: Universidad de San Buenaventura. Psycología. Avances de la disciplina, vol 2, núm 1, pp. 133-160.

Planas, N. y Alsina, Á. (2009). Educación matemática y buenas prácticas: Infantil, primaria secundaria y educación superior. Barcelona-España: Graó. Biblioteca del Aula.

Pozo, I. y Gómez, M. (2001). Aprender y enseñar ciencia. Madrid-España: Morata.

Toro, I. y Parra, R. (2010). Fundamentos epistemológicos de la investigación y la metodología de la investigación cualitativa/cuantitativa. Medellín-Colombia: Fondo Editorial Universidad EAFIT.

Vásquez, A. y Manassero, M. (2001). Opiniones sobre las relaciones entre ciencia, tecnología y sociedad. Madrid: Tarbiya, revista de Investigación e Innovación Educativa, vol 27, pp. 27-56. 